\title{
Gravitational force between two electrons in superconductors
}

\author{
Clovis Jacinto de Matos*
}

October 31, 2018

\begin{abstract}
The attractive gravitational force between two electrons in superconductors is deduced from the Eddington-Dirac large number relation, together with Beck and Mackey electromagnetic model of vacuum energy in superconductors. This force is estimated to be weaker than the gravitational attraction between two electrons in the vacuum.
\end{abstract}

\section{Introduction}

Suspicions about the underlying physical connections among the parameters of the cosmos and particle physics arose as early as 1930 when Eddington [1] and Dirac 22 investigated myriad occurrences of pure numbers of order $10^{-40}$. These relations can be summarized in the following expression [3]:

$$
\alpha\left(\frac{m_{p}}{m}\right)=\left(\frac{\Lambda^{-1 / 2}}{l_{p}}\right)^{1 / 3} .
$$

Where $l_{p}=\left(\frac{G \hbar}{c^{3}}\right)^{1 / 2}$ is the Planck length, $m_{P}=\left(\frac{\hbar c}{G}\right)^{1 / 2}$ is the Planck mass, $m$ is the electron mass, $\alpha=\frac{1}{4 \pi \epsilon_{0}} \frac{e^{2}}{\hbar c}$ is the fine structure constant,and $\Lambda$ is the cosmological constant. Using the presently measured value of $\Lambda_{0}=$ $(1.29 \pm 0.23) \times 10^{-52}\left[\mathrm{~m}^{-2}\right]$ [4], Equ.(1) is valid within $1 \%$.

A modern formulation of the Eddington-Dirac large number conjecture, equ.(1), is possible in the framework of the Planck-Einstein scale [5, 6], which

*ESA-HQ, European Space Agency, 8-10 rue Mario Nikis, 75015 Paris, France, e-mail: Clovis.de.Matos@esa.int 
corresponds to typical energy scales for dark energy, and involves the fundamental constants: $\Lambda, \hbar, c, k, G$. Explicitly one has the following formulas at the Planck-Einstein scale:

$$
\begin{gathered}
E_{P E}=k T_{P E}=\left(\frac{c^{7} \hbar^{3} \Lambda}{G}\right)^{1 / 4}=5.25[\mathrm{meV}] \\
m_{P E}=\frac{E_{P E}}{c^{2}}=\left(\frac{\hbar^{3} \Lambda}{c G}\right)^{1 / 4}=9.32 \times 10^{-39}[\mathrm{Kg}] \\
l_{P E}=\frac{\hbar}{M_{P E} c}=\left(\frac{\hbar G}{c^{3} \Lambda}\right)^{1 / 4}=0.037[\mathrm{~mm}] \\
t_{P E}=\frac{l_{P E}}{c}=\left(\frac{\hbar G}{c^{7} \Lambda}\right)^{1 / 4}=1.26 \times 10^{-13}[\mathrm{~s}] \\
\rho_{P E}=\frac{E_{P E}}{l_{P E}^{3}}=\frac{c^{4} \Lambda}{G}=104\left[\mathrm{eV} / \mathrm{mm}^{3}\right]
\end{gathered}
$$

The Planck temperature $T_{P}=\frac{1}{k} \sqrt{\frac{\hbar c^{5}}{G}}$ together with the Planck-Einstein temperature, $T_{P E}=\frac{1}{k}\left(\frac{c^{7} \hbar^{3} \Lambda}{G}\right)^{1 / 4}$, allow to rewrite equ.(1) in the following form:

$$
\alpha\left(\frac{m_{P}}{m}\right)=\left(\frac{T_{P}}{T_{P E}}\right)^{2 / 3} .
$$

Thus it seems that the Eddington-Dirac large number relation takes its "natural form" in the context of the Planck-Einstein scale.

The existence of dark energy in the universe, as indicated by numerous astrophysical observations, represents one of the most challenging problems in theoretical physics at present [4, 7, 8, 9]. A great variety of different models exist for dark energy but none of these models can be regarded as being entirely convincing so far. The cosmological constant problem (i.e. the smallness of the cosmologically observed vacuum energy density) remains an unsolved problem. It is likely that the solution of this problem requires new, so far unknown, physics.

Recent models of dark energy, such as the electromagnetic dark energy model of Beck and Mackey [10, produce potentially measurable effects at laboratory scales, which are, however, restricted to superconductors. In [10] a Ginzburg-Landau theory is constructed that generates a cutoff for the gravitational activity of vacuum fluctuations. Generally it is assumed in this model that vacuum fluctuations of any particle can exist in two different 
phases: A gravitational active one (contributing to the cosmological constant $\Lambda$ ) and a gravitationally inactive one (not contributing to $\Lambda$ ). The model exhibits a phase transition at a critical frequency which makes the dark energy density in the universe small and finite. The above approach has many analogies with the physics of superconductors, and in particular it allows for a possible interaction between dark energy and Cooper pairs. Using the vacuum energy predicted by this model in superconducting materials together with the Eddington-dirac conjectured relationship equ.(1), we estimate the gravitational force between two electrons in superconducting materials, and compare it with its value in vacuum.

\section{Electron's gravito-electromagnetic coupling from the Eddington-Dirac large number re- lation}

From Eddington-Dirac large number relation, Equ.(1), we deduce the coupling between the gravitational and the electromagnetic interaction between two electrons in vacuum, in function of the electron Compton wavelength $\lambda_{c}=\frac{\hbar}{m c}$, the cosmological constant, and the square of the fine structure constant, $\alpha$

$$
\frac{\alpha_{g}}{\alpha}=\alpha^{2} \lambda_{c} \Lambda^{1 / 2}
$$

Where $\alpha_{g}=\frac{G m^{2}}{\hbar c}=\left(\frac{m}{m_{P}}\right)^{2}$ is the electron's gravitational fine structure constant. Note that a null value of the cosmological constant would imply that electrons could note simultaneously generate gravitational and electromagnetic fields.

A non-vanishing cosmological constant (CC) $\Lambda$ can be interpreted in terms of a non-vanishing vacuum energy density

$$
\rho_{v a c \Lambda}=\frac{c^{4}}{8 \pi G} \Lambda
$$

which corresponds to dark energy. The small astronomically observed value of the CC, $\Lambda=1.29 \times 10^{-52}\left[1 / \mathrm{m}^{2}\right]$ [4], and its origin remain a deep mystery. This is often call the CC problem, since with a cutoff at the Planck scale the vacuum energy density expected from quantum field theories should be larger by a factor of the order $10^{120}$, in complete contradiction with the observed value. Substituting equ(9) into equ.(8), it is possible to express the coupling 
between gravitational and electromagnetic forces between two electrons in vacuum in function of the dark energy density.

$$
\frac{\alpha_{g}}{\alpha}=\frac{2(2 \pi G)^{1 / 2}}{c^{2}} \alpha^{2} \lambda_{c} \rho_{v a c \Lambda}^{1 / 2}
$$

\section{Electromagnetic model of vacuum energy in superconductors}

To solve the CC problem, in [10] a model of dark energy was suggested that is based on electromagnetic vacuum fluctuations creating a small amount of vacuum energy density. One assumes that photons (or any other bosons), with zeropoint energy $\epsilon=\frac{1}{2} h \nu$, can exist in two different phases: A gravitationally active phase where the zeropoint fluctuations contribute to the cosmological constant $\Lambda$, and a gravitationally inactive phase where they do not contribute to $\Lambda$ [10, 11, 12, 13]. This is described in [10] by a GinzburgLandau type of theory. As shown in [10], this type of model of dark energy can lead to measurable effects in superconductors, via a possible interaction with the Cooper pairs in the superconductor.

Here we introduce an additional hypotheses with respect to the original Beck and Mackey model:

1. the vacuum energy density contained in superconductors can be different from the energy density observed in the universe.

Beck and Mackey's Ginzburg-Landau-like theory leads to a finite dark energy density dependent on the frequency cutoff $\nu_{c}$ of vacuum fluctuations:

$$
\rho^{*}=\frac{1}{2} \frac{\pi h}{c^{3}} \nu_{c}^{4}
$$

In vacuum one may put $\rho^{*}=\rho_{v a c \Lambda}$, from which the cosmological cutoff frequency $\nu_{c c}$ is estimated as

$$
\nu_{c c} \simeq 2.01 T H z
$$

The corresponding "cosmological" quantum of energy is:

$$
\epsilon_{c c}=h \nu_{c c}=8.32 \mathrm{meV}
$$

In the interior of superconductors, according to assumption 1 . above, the effective cutoff frequency can be different. This is due to interaction effects between the two Ginzburg-Landau potentials (that of the superconducting 
electrons and that of the dark energy model) [10. The effect can be seen in analogy to polarization effects of ordinary electromagnetic fields in matter: In matter the electric field energy density is different as compared to the vacuum. Similarly, in superconductors the effectice dark energy density (represented by gravitationally active zeropoint fluctuations) can be different as compared to the vacuum. Our model allows for the gravitational analogue of polarization.

An experimental effort is currently taking place at University College London and the University of Cambridge to measure the cosmological cutoff frequency through the measurement of the spectral density of the noise current in resistively shunted Josephson junctions, extending earlier measurements of Koch et al. [14].

In [10] the formal attribution of a temperature $T$ to the graviphotons is done by comparing their zeropoint energy with the energy of ordinary photons in a bath at temperature $T$ :

$$
\frac{1}{2} h \nu=\frac{h \nu}{e^{\frac{h \nu}{k T}}-1}
$$

This condition is equivalent to

$$
h \nu=\ln 3 k T
$$

Substituting the critical transition temperature $T_{c}$ specific to a given superconductive material into Eq.(15), we can calculate the critical frequency characteristic for this material:

$$
\nu_{c}=\ln 3 \frac{k T_{c}}{h}
$$

For example, for Niobium with $T_{c}=9.25 \mathrm{~K}$ we get $\nu_{c}=0.212 \mathrm{THz}$. If we use the cosmological cutoff frequency, equ.(12), in Eq.(16) we find the cosmological critical temperature $T_{c c}$ :

$$
T_{c c}=87.49 \mathrm{~K}
$$

This temperature is characteristic of the BSCCO High- $T_{c}$ superconductor.

Substituting equ.(16) into equ.(11), the vacuum energy stored in a given superconductor is obtained from a Stephan-Boltzmann type law, showing a dependence on the fourth power of the superconductor's critical transition temperature:

$$
\rho^{*}=\frac{\pi(\ln 3)^{4}}{2} \frac{k^{4}}{(c h)^{3}} T_{c}^{4}
$$




\section{The gravitational force law between two electrons in superconductors}

Using Beck and Mackey's electromagnetic vacuum energy density in superconductors $\rho^{*}$, equ.(18), instead of the cosmological energy density $\rho_{\text {vac } \Lambda}$, equ.(9), in the expression defining the coupling between gravitational and electromagnetic forces between two electrons in vacuum, equ.(10), we obtain this coupling in a superconducting environment:

$$
\frac{\alpha_{g}}{\alpha}=\alpha^{2} \frac{(\ln 3)^{2}}{(2 \pi)^{1 / 2}} \frac{k^{2}}{m m_{P} c^{4}} T_{c}^{2}
$$

Taking the geometric mean value between the Planck scale and the electron scale, it is possible to define an intermediate energy scale at $7.9[\mathrm{GeV}]$ :

$$
m_{i}=\left(m m_{P}\right)^{1 / 2}
$$

substituting equ.(20) into equ.(19), we obtain:

$$
\frac{\alpha_{g}}{\alpha}=\alpha^{2} \frac{(\ln 3)^{2}}{(2 \pi)^{1 / 2}}\left(\frac{T_{c}}{T_{i}}\right)^{2}
$$

where $T_{i}=m_{i} c^{2} / k \sim 9 \times 10^{20}[K]$ is the temperature associated with the intermediate energy scale defined from equ.(20)

Equ.(21) indicates that the coupling between gravitational forces and electromagnetic forces between two electrons in superconductors is weaker than in vacuum, recovering its classical value at the temperature, $T_{c} \sim 87.5[\mathrm{~K}]$ (BSCCO's critical transition temperature).

Assuming that the electromagnetic fine structure constant does not change, with respect to its classical value. We can only explain a deviation from the classical gravito-electromagnetic coupling in terms of a suitable scale transformation: The gravitational constant $G=\hbar c / m_{P}^{2}$ formally becomes much weaker in a superconductor than in vacuum if $m_{P}$ is replaced by a higher value $m_{P e f f}$. Thus from equ.(21) we deduce the effective gravitational constant between two electrons in a given superconducting material:

$$
G_{\text {eff }}=\frac{\hbar c}{m_{\text {Peff }}^{2}}=\frac{\alpha^{2}}{4 \pi \epsilon_{0}} \frac{(\ln 3)^{2}}{(2 \pi)^{1 / 2}}\left(\frac{e}{m}\right)^{2}\left(\frac{T_{c}}{T_{i}}\right)^{2}
$$

Substituting this expression in Newton's law of gravitation, and applying it to a system of two electrons separated by a distance $r$ inside a superconductor. We conclude that their mutual gravitational attraction force, $F_{S C}$, will 
be smaller than the gravitational force, $F_{0}$, that the same electrons, separated by the same distance, will exert on each other when they are in vacuum and the classical value of the gravitational constant $G_{0}=6.67 \times 10^{-11}\left[\mathrm{Nm}^{2} / \mathrm{Kg}^{2}\right]$ holds:

$$
\frac{F_{S C}}{F_{0}}=\frac{G_{e f f}}{G_{0}}=\frac{\alpha^{2}}{4 \pi \epsilon_{0} G_{0}} \frac{(\ln 3)^{2}}{(2 \pi)^{1 / 2}}\left(\frac{e}{m}\right)^{2}\left(\frac{T_{c}}{T_{i}}\right)^{2}
$$

For the case of two electrons located in superconducting Niobium, for which $T_{c}=9.25[K]$, the numerical estimation of equ.(23) is:

$$
\frac{F_{S C}}{F_{0}} \sim 10^{-2}
$$

This prediction is difficult to test experimentally, since the electron's mass only contributes marginally to the overall mass of atoms and of macroscopic bodies: $\left(m_{\text {electron }} / m_{\text {proton }}=5.4 \times 10^{-4}\right)$.

At this point one remark is in order: Our theoretical derivation presented in this paper strictly speaking holds only for electrons, because equ.(1) is only valid for electrons (in that expression $m$ must always be the electron mass). Therefore equ.(23) should not apply to the other particles (protons, atoms) the superconductor consists of. This ultimately could be understood as being a direct consequence of the spontaneous breaking of the principle of general covariance in superconductors [15].

\section{5 conclusion}

From the Eddington-Dirac Large number conjecture, equ.(1), together with Beck and Mackey electromagnetic model for dark energy density in superconductors, equ.(18), it is shown that the gravitational force between two electrons in superconductors should deviate from the classical law of gravitational attraction between two electrons in vacuum, according to equ.(23). This effect is estimated difficult to be detected within current experimental capabilities.

\section{Acknowledgment}

The author is grateful to Prof. Christian Beck for useful comments on the physical interpretation of the proposed conjecture. 


\section{References}

[1] Eddington, A.S., "Proceedings of the Cambridge Philosophical Society", 27,15 (1931).

[2] Dirac, P.A.M., NAture, 139, 323 (1937)

[3] Nottale, L., "Scale Relativistic Cosmology", Chaos, Solitons and Fractals, 16, 539, (2003)

[4] Spergel, D.N., et al., Astrophy. J. Suppl. 148, 175 (2003) and references therein

[5] Beck, C., de Matos, C.J., "The dark energy scale in superconductors: Innovative theoretical and experimental concepts" to appear in Found. Phys. (2007), arXiv:0709.2373

[6] de Matos, C.J., "Electromagnetic Dark Energy and Gravitoelectrodynamics of superocnductors", to appear in Physica C (2007) arXive:0704.2499

[7] P. J. E. Peebles, B. Ratra, Rev. Mod. Phys. 75, 559 (2003)

[8] E.J. Copeland, M. Sami, and S. Tsujikawa, Int. J. Mod. Phys. D 15, 1753 (2006)

[9] T. Padmanabhan, Phys. Rep. 380, 235 (2005)

[10] C. Beck, M. C. Mackey, "Electromagnetic Dark Energy", astro-ph/0703364

[11] C. Beck, M. C. Mackey, Phys. Lett. B 605, 295 (2005).

[12] C. Beck, J.Phys.Conf.Ser. 31, 123 (2006).

[13] C. Beck, M. C. Mackey, Physica A 379, 101 (2007)

[14] R. H. Koch, D. van Harlingen, and J. Clarke, Phys. Rev. Lett. 45, 2132 (1980)

[15] de Matos, C.J., Beck, C., "Possible measurable effects of dark energy in rotating superconductors" to appear in Class. Quant. Grav. (2007), arXiv:0707:1797 